\title{
Translational gaps in animal models of human infusion reactions to nanomedicines
}

\author{
S Moein Moghimi* ${ }^{* 1,2,3}$ \& Dmitri Simberg ${ }^{3}$ \\ ${ }^{1}$ School of Pharmacy, The Faculty of Medical Sciences, King George VI Building, Newcastle University, Newcastle upon Tyne, NE1 \\ 7RU, UK \\ ${ }^{2}$ Division of Stratified Medicine, Biomarkers \& Therapeutics, Institute of Cellular Medicine, Newcastle University, Framlington Place, \\ Newcastle upon Tyne, NE2 4HH, UK \\ ${ }^{3}$ Translational Bio-Nanosciences Laboratory \& Colorado Center for Nanomedicine \& Nanosafety, The Skaggs School of Pharmacy \& \\ Pharmaceutical Sciences, Department of Pharmaceutical Sciences, University of Colorado Denver, Anschutz Medical Campus, 1250 \\ East Mountview Blvd, Aurora, CO 80045, USA \\ *Author for correspondence: seyed.moghimi@ncl.ac.uk
}

"we question limitations and suitability of the porcine model in assessing infusion reactions to nanomedicines"

Adverse infusion reactions to regulatory approved nanomedicines in human subjects are idiosyncratic, but outwardly reproducible in pigs. A large body of evidence suggests that the porcine reactions are related to robust nanoparticle clearance by pulmonary intravascular macrophages (PIMs), and rapid release of arachidonate metabolites from these cells. Similar to pigs, other animals that have resident PIMs in their lungs also respond to intravenously injected particles, where rapid particle clearance by PIMs correlate with peak periods of cardiopulmonary distress. Normal human lungs, however, do not have PIMs, but 'induced' PIMs have been identified in pulmonary circulation under certain pathological conditions. We question suitability, and limitation of these preclinical models for global assessment of nanomedicine safety, and discuss alternative models and approaches.

First draft submitted: 27 February 2018; Accepted for publication: 28 February 2018; Published online: 23 May 2018

Keywords: adverse reactions $\bullet$ nanomedicine $\bullet$ nanoparticle $\bullet$ pig $\bullet$ pulmonary intravascular macrophages $\bullet$ sheep

Mucocutaneous responses (e.g., erythema, flushing, rash, rhinitis, tearing) and cardiopulmonary distress (e.g., arrhythmia, edema, hypertension, hyperventilation, hypotension, hypoxia, tachycardia, shortness of breath) are among the most frequent symptoms observed on nanomedicine infusion to sensitive individuals [1]. These idiosyncratic reactions are not true allergy, as they are not mediated by pre-existing allergen-reactive IgEs [1]. These reactions, including some hematological changes, however, are outwardly reproducible in pigs, and as such the porcine model has been suggested for evaluating the safety (infusion reactions) of nanopharmaceuticals under development [2]. Here, from a pathophysiological perspective, we question limitations and suitability of the porcine model in assessing infusion reactions to nanomedicines, and call for alternative approaches.

Morphological studies have confirmed the presence of resident macrophages in the pulmonary vessels of pigs as well as other species such as sheep, calves, goats and cats, but not in humans and laboratory animals such as mice and rats [3]. These macrophages, termed pulmonary intravascular macrophages (PIMs), have long been shown to rapidly extract particulate materials of different sizes and characteristics from the blood [3], whereas in other species (e.g., mouse, rat, human) the hepatic Kupffer cells and splenic macrophages predominantly intercept and clear blood-borne particles [4]. For examples, in pigs $90 \%$ of intravenously injected air-filled albumin microspheres (Albunex ${ }^{\circledR}$, Molecular Biosystems, CA, USA) was recovered in the lungs, whereas in rats hepato-splenic sites where the major site of Albunex deposition, and uptake by the lungs accounted for $5 \%$ of the injected dose [5]. A detailed biodistribution study by Brain et al. [6] in different species have also shown predominant deposition of intravenously injected gold and iron oxide nanoparticles to the lungs of sheep, calves, pigs and cats, but not in monkeys, rabbits, guinea pigs, rats, mice and chickens. Another study has shown the ability of sheep PIMs to clear substantial amounts 
of intravenously injected liposomes ( $\sim 60 \%$ of the dose within $2 \mathrm{~h}$ of injection) [7]. Here, rapid clearance of liposomes by PIMs not only correlated with peak periods of cardiopulmonary distress (e.g., vasoconstriction, broncoconstriction and pulmonary hypertension), but also PIMs were the prime source of the arachidonate metabolites rather than the endothelial cells, neutrophils or perivascular interstitial cells [7]. Furthermore, pulmonary hemodynamic responses were completely prevented by cyclooxygenase inhibitor indomethacin [7]. These observations mirror our own studies and others $[3,8,9]$, where PIM destruction in pigs (through administration of clodronate-encapsulated liposomes) abrogated thromboxane release, and hence cardiopulmonary distress on injection of various particulate agents (e.g., polystyrene nanoparticles and PEGylated liposomes, zymosan), and endotoxin. Similarly, newborn lambs do not have PIMs, and do not undergo cardiopulmonary distress on particle injection [10]. However, within 2 weeks of birth, PIMs appear in lamb lungs, and particle injection induces a transient pulmonary hypertension [10]. Since, a population of PIMs are believed to be the likely source of thromboxane, and the fact that pulmonary hemodynamic and lymph dynamic changes occur in a dose-dependent fashion to particle injection [3,7-9], testing of nanomedicine safety in porcine (and other ruminants) will most likely induces cardiopulmonary distress. Accordingly, such global outcomes may exclude otherwise, promising nanopharmaceuticals from the development pipeline on safety grounds that are not relevant to wider human populations [1,11]. In line with this argument, it is worth noting that both pigs and adult sheep also show transient pulmonary hypertension on injection/infusion of small doses of Intralipid ${ }^{\circledR}$ (a 20\% iv. fat emulsion approved for human use; Fresenivs Kabi, Uppsala, Sweden), whereas infusion reactions to Intralipid in humans rarely occur [12,13]. Same observation applies to Albunex, which has also been approved for human use [11,14]. Therefore, global nanomedicine safety assessment in the porcine model (and other ruminants and species with PIMs) is perhaps inappropriate and misleading [1,11], and therefore these models should not be advertently promoted and their applications exaggerated [15].

On the other hand, a potential utility of the pig and sheep models could be that they represent an extreme situation such as the human adult respiratory distress syndrome, where 'induced' PIMs are abundant in the lungs [3]. Nevertheless, the porcine model has proved useful in identifying physicochemical characteristics of nanoparticles that overcome macrophage responses. For example, changing particle shape from oblate spheroids to rods and disks (with appropriate aspect ratios), transiently delays robust particle clearance by PIMs on intravenous injection, and this overcomes cardiopulmonary distress [8]. Furthermore, injection reactions to oblate spheroids in pigs are also dampened on erythrocyte attachment prior to administration [8]. These studies indicate that slowing the rate of particle presentation to PIMs may trigger desensitization. These observations are in agreement with clinical studies, where slow infusion rates have in many cases overcome cardiopulmonary distress [1]. On the basis of these observations we have also shown that reducing the size of oblate spheroids (e.g., to dimensions below $100 \mathrm{~nm}$, and with volumes similar to nonreactive rods and disks) or decreasing the particle dose (even with larger sizes), can also dampen injection reactions in pigs regardless of particle surface chemistry (Wibroe \& Moghimi 2016, University of Copenhagen, Unpublished observations).

We argue that better models can, and should be developed through improved understanding of mechanism of hypersensitivity in humans, and based on physiological and immunological drivers of these reactions. However, 'induced' PIMs appear in pulmonary circulation of patients with liver dysfunction and disease, hepatopulmonary syndrome and inflammatory lung disease [1]. Other responsive cells may include lung dendritic cells and certain subpopulation of macrophages in the liver, spleen and skin in such patients, but not investigated. Nevertheless, biopsies or autopsied lungs from such patients may provide valuable sources for understanding the biology of constitutive or 'induced' PIMs, including receptors that trigger adverse responses, and signaling pathways that start desensitization processes. Lack of injection reactions to nanomedicines in pigs or sheep (as in rods and disks) may not necessarily translate to safety in humans, since human macrophages may respond differently within their own microenvironment.

Further development of improved animal models of human injection/infusion reactions, however, should still consider the fact that PIMs are not natural resident in human pulmonary circulation. At the same time, any animal model would be of limited use if based only on phenotypical correlation rather than on physiological mechanism. Until then, it would be risky to claim the applicability of any given animal model to predict infusion-related toxicities of newly developed nanomedicines in human patients. On the basis of these arguments, the cirrhotic rat model might offer a reasonable starting point, where in contrast to normal rats, a population of large macrophages can be seen in the pulmonary circulation [16]. Here, with disease progression, Kupffer cells show a decrease tendency in extracting intravenously injected particles from the blood, whereas particle deposition to the lungs increases (due to rapid clearance by induced macrophages in pulmonary circulation). By controlling the extent of biliary cirrhosis 
(and hence the positioning and number of induced PIMs), the rat model may set the ground for representing mild, moderate and severe cases of human reactions in assessing/predicting nanomedicine injection/infusion safety.

\section{Financial \& competing interests disclosure}

The authors have no relevant affiliations or financial involvement with any organization or entity with a financial interest in or financial conflict with the subject matter or materials discussed in the manuscript. This includes employment, consultancies, honoraria, stock ownership or options, expert testimony, grants or patents received or pending, or royalties.

No writing assistance was utilized in the production of this manuscript.

\section{References}

1. Moghimi SM. Nanomedicine safety in preclinical and clinical development: focus on idiosyncratic injection/infusion reactions. Drug Discov. Today doi:10.1016/j.drudis.2017.11.006 (2017) (Epub ahead of print).

2. Szebeni J, Bedocs P, Csukas D, Rosivall L, Bunger R, Urbanics R. A porcine model of complement-mediated infusion reactions to drug carrier nanosystems and other medicines. Adv. Drug Deliv. Rev. 64, 1706-1716 (2012).

3. Schneberger D, Aharonson-Raz K, Singh B. Pulmonary intravascular macrophages and lung health: what are we missing? Am. J. Physiol. Lung Cell Mol. Physiol. 302, L498-L503 (2012).

4. Moghimi SM, Hunter AC, Andresen TL. Factors controlling nanoparticle pharmacokinetics: an integrated analysis and perspective. Annu. Rev. Pharmacol. Toxicol. 52, 481-503 (2012).

5. Walday P, Tolleshaug H, Gjøen T et al. Biodistributions of air-filled albumin microspheres in rats and pigs. Biochem. J. 299, 437-443 (1994).

6. Brain JD, Molina RM, DeCamp MM, Warner AE. Pulmonary intravascular macrophages: their contribution to the mononuclear phagocytic system in 13 species. Am. J. Physiol. 276, L146-L154 (1999).

7. Miyamoto K, Schultz E, Heath T, Mitchell MD, Albertine KH, Staub NC. Pulmonary intravascular macrophages and hemodynamic effects of liposomes in sheep. J. Appl. Physiol. 64, 1143-1152 (1988).

8. Wibroe PP, Anselmo A, Nilsson PH et al. Bypassing adverse injection reactions to nanoparticles through shape modification and attachment to erythrocytes. Nat. Nanotechnol. 12, 589-594 (2017).

9. Gaca JG, Palestrant D, Lukes DJ, Olausson M, Parker W, Davis RD Jr. Prevention of acute lung injury in swine: depletion of pulmonary intravascular macrophages using liposomal clodronate. J. Surg. Res. 112, 19-25 (2003).

10. Longworth KE, Westgate AM, Grady MK, Westcott JY, Staub NC. Development of pulmonary intravascular macrophage function in newborn lambs. J. Appl. Physiol. 73, 2608-2615 (1992).

11. Skotland T. Injection of nanoparticles into clover-hoof animals: asking for trouble. Theranostics 7, 4877-4878 (2017).

12. Mckeen CR, Brigham KL, Bowers RE, Harris TR. Pulmonary vascular effects of fat emulsion infusion in unanesthetized sheep. J. Clin. Invest. 61, 1291-1297 (1978).

13. Bedocs $\mathrm{P}$, Capacchione J, Potts $\mathrm{L}$ et al. Hypersensitivity reactions to intravenous lipid emulsion in swine: relevance for lipid resuscitation studies. Anesth. Analg. 119, 1094-1101 (2014).

14. Østensen J, Hede R, Myreng Y, Ege T, Holtz E. Intravenous injection of Albunex ${ }^{\circledR}$ microspheres causes thromboxane mediated pulmonary hypertension in pigs, but not in monkeys or rabbits. Acta Physiol. Scand. 144, 307-315 (1992).

15. Szebeni J, Storm G. Complement activation as a bioequivalence issue relevant to the development of generic liposomes and other nanoparticulate drugs. Biochem. Biophys. Res. Commun. 468, 490-497 (2015).

16. Mit-Noirault E, Faure L, Guichard Y, Montharu J, Le Pape A. Scintigraphic in vivo assessment of the development of pulmonary intravascular macrophages in liver disease. Chest 120, 941-947 (2001). 